\title{
Diagnostic accuracy of the risk of malignancy index 1 compared to the more recent IOTA ADNEX model in discriminating benign from malignant adnexal masses: a multi-centric study
}

\author{
Amita Ray ${ }^{1 *}$, Divya S. ${ }^{2}$, B. N. Kumar Guru ${ }^{3}$, A. S. Ramaswamy ${ }^{3}$, Bharat Kumar ${ }^{4}$
}

\begin{abstract}
${ }^{1}$ Department of Obstetrics and Gynecology, IQ City Medical College, Durgapur, West Bengal, India
${ }^{2}$ Department of Obstetrics and Gynecology, DM Wayanad Institute of Medical Sciences, Wayanad, Kerala, India

${ }^{3}$ Department of Pathology PES, Institute of Medical Sciences and Research, Kuppam, Andhra Pradesh, India

${ }^{4}$ Statistician, GSK Pharmaceuticals, Bangalore, Karnataka, India
\end{abstract}

Received: 04 January 2019

Accepted: 05 February 2019

\section{*Correspondence:}

Dr. Amita Ray,

E-mail: amitarays@gmail.com

Copyright: () the author(s), publisher and licensee Medip Academy. This is an open-access article distributed under the terms of the Creative Commons Attribution Non-Commercial License, which permits unrestricted non-commercial use, distribution, and reproduction in any medium, provided the original work is properly cited.

\begin{abstract}
Background: Identification of the nature of an adnexal mass can ensure optimum management. Single parameters as well as diagnostic models using a combination of several parameters are in use. The International Ovarian Tumor Analysis (IOTA) consortium has developed and published the Assessment of Different NEoplasias in the adneXa (ADNEX) model, which differentiates between benign and malignant masses. Authors conducted this study with the aim of finding a cut off value for this model in the study population and comparing the diagnostic accuracy of this model to that of the risk of malignancy (RMI).

Methods: Women with adnexal masses admitted to the 3 medical college affiliated hospitals for surgical management were included in this study. Appropriate investigations were done to calculate the RMI-I and ADNEX score for each participant. A cut off score for the ADNEX model was determined and diagnostic accuracy tests were done for comparison.

Results: At a cut-off of 29 for the ADNEX model and 200 for RMI model the sensitivity was 75\% and 77.8, specificity 100\% and 80.6\%; Positive Predictive Value (PPV) 100\%and 60\%; Negative Predictive Value (NPV) $91 \%$ and 90.6\%; Positive Likelihood ratio of infinity and 4 and a negative Likelihood Ratio of 2.8 and 2.5 respectively.

Conclusions: The ADNEX model rates higher than the RMI in almost all tests of diagnostic accuracy and can be used for triaging, framing a referral policy and prioritizing surgery.
\end{abstract}

Keywords: Adnexal mass, IOTA ADNEX model, Risk of Malignancy Index (RMI), Tests of diagnostic accuracy

\section{INTRODUCTION}

Ovarian masses are one of the most common problems faced by a practicing gynecologist. Single parameters as well as diagnostic models using a combination of several parameters are in use to distinguish benign from malignant. ${ }^{1-4}$ The Royal College of Obstetrics and Gynecologist (RCOG) recommends RMI I as a validated tool to distinguish benign from malignant masses. A cut off of 200 with a sensitivity of $78 \%$ and a specificity of $87 \%$, or a cut off of 250 with a lower sensitivity $(70 \%)$ but higher specificity $(90 \%)$ are equally good to plan management The American College of Obstetricians and Gynecologists (ACOG) in its guidelines advocates referring the patient to an oncology centre if the CA-125 is greater than 35 in a post-menopausal woman and greater than 200 in a premenopausal woman. ${ }^{5,6}$ Recently the International Ovarian Tumor Analysis (IOTA) 
consortium has developed and published the Assessment of Different Neoplasias in the adnexa (ADNEX) model, which differentiates between benign and four types of malignancy namely borderline, stage I cancer, stage II-IV cancer, and secondary metastatic cancer. As with other models it suggests that every clinical setting should define their own cut offs for management as well as referrals. ${ }^{7}$ Knowing the nature of the mass goes a long way in the preparedness for dealing with it. Whether they should be operated, who should operate, where and how the operation needs to be done and how the patient and her attendants are to be counselled are important questions that need answers. ${ }^{1}$ This combined with the fact that the cut off for diagnostic models should be determined as per the clinical setting authors conducted this study with the aim of finding a cut off for the IOTA model in present study population and comparing the diagnostic accuracy of this model with that of the RMI-1 model which authors had been being used for planning the management of adnexal masses. The objectives of the present study are determining a cut off for the IOTA ADNEX model, to distinguish between benign and malignant adnexal masses in the study population and comparing the diagnostic accuracy of RMI 1 with the IOTA ADNEX model using the above cut off.

\section{METHODS}

\section{Data collection}

Women with adnexal masses admitted to the 3-medical college affiliated hospitals for surgical management were included in this study. Women with adnexal masses who were either managed conservatively, were not fit for surgery or denied surgery were excluded. Informed consent (Appendix-1) was obtained from all participants.

Transvaginal ultrasound was performed and if the mass was too big to be observed completely a trans-abdominal scan was also done. Serum CA-125 levels were measured using Electro-Chemi-LuminescenceImmuno-Assay (ECLIA). Based on the data collected the RMI-1 Score and IOTA ADNEX percentage for each participant was calculated (Appendix-2 and Appendix-3 respectively).

Using Histopathology as the reference standard, the data analysis was done in two parts:

- Finding the cut off for the IOTA model using a ROC curve.

- Comparing the RMI model with the IOTA using different tests of diagnostic accuracy

\section{Choosing a cut off for the ADNEX model}

Using the sensitivities and specificities of the IOTA ADNEX an ROC curve was plotted (Appendix 4). Area Under Curve (AUC) value 0.978 ( $\mathrm{p}$ value $<0.001$ ) shows that IOTA has a very good predictive ability to discriminate benign from malignant adnexal masses.

For present study authors chose the cut off value as 29.29 which had a sensitivity of $75 \%$ and a specificity of $100 \%$. This is quite comparable to the sensitivity and specificity values for the RMI cut off of 200.

Table 1: For analysis of the data.

\begin{tabular}{|l|l|l|l|l|}
\hline \multicolumn{5}{|c|}{ The gold standard test (histopathology) } \\
The test to be \\
\cline { 2 - 5 } $\begin{array}{l}\text { assessed } \\
\text { (RMI or IOTA) }\end{array}$ & Positive (Number) & A (true positive) & B (false positive) & Test positive \\
\hline & Negative (Number) & C (true negative) & D (false negative) & Test negative \\
\hline & T (disease) & T (non-disease) & Total
\end{tabular}

Sensitivity $=(\mathrm{a} / \mathrm{a}+\mathrm{c}) \times 100$, Specificity $=(\mathrm{d} / \mathrm{b}+\mathrm{d}) \times 100$, Positive predictive value $=(\mathrm{a} / \mathrm{a}+\mathrm{b}) \times 100$, Negative predictive value $=(\mathrm{d} / \mathrm{c}+\mathrm{d})$ x100, Positive Likelihood Ratio = Sensitivity / 1-Specificity, Negative Likelihood Ratio=1-Sensitivity/Specificity, Diagnostic accuracy $=(\mathrm{a}+\mathrm{d}) /$ Total

Thus, participants having a risk calculation of 29 and above were taken as having malignant masses and those with calculation below 29 were taken to be benign..$^{5}$ The centers where this study was done are not oncology centers. Suspected malignancies requiring surgery are referred to an oncology centre where the management is done by a multidisciplinary team. One of the main reasons why the study was done was to improve triaging, referrals and prioritizing surgeries. The 29 cut off has the maximum sensitivity for $100 \%$ specificity. Comparing the RMI model with the IOTA using different tests of diagnostic accuracy. There are several measures that relate to the different aspects of the diagnostic procedure. For present study authors calculated the sensitivity, specificity, positive and negative likelihood ratios, positive predictive value (PPV) and negative predictive value (NPV) and diagnostic accuracy. Discriminatory tests like sensitivity and specificity are not affected by disease prevalence whereas tests used to assess the predictive ability are affected by disease prevalence. ${ }^{8,9}$ Sensitivity, specificity and the likelihood ratios are methods that can be used to frame policies for triaging patients. Since these values are not affected by disease prevalence they can also be extrapolated to other 
populations. ${ }^{10,11} \mathrm{PPV}, \mathrm{NPV}$ and diagnostic accuracy are predictive methods which are useful in predicting the probability of malignancy in an individual case and could help the clinician in counseling the woman and her family. Since these values are dependent on disease prevalence, they are useful in knowing the probability of malignancy in a particular individual and are not applicable to other settings. In this study of diagnostic accuracy, authors have also included all the items listed in STARD: Standards for Reporting Diagnostic accuracy studies. (Appendix-5) This list of items was developed to contribute to the completeness and transparency of diagnostic accuracy studies..$^{10,11}$

\section{RESULTS}

The mean age of the participants was 44 years with a Standard Deviation (SD) of 14.86 (Table 2). Using
Histopathology as the gold standard for diagnosing malignancy the RMI had 21 true positives (a), 14 false positives (b), 6 true negatives (c) and 58 false negatives (d) (Table 3).

Table 2: Age distribution.

\begin{tabular}{|l|l|l|}
\hline & Mean & Standard Deviation (SD) \\
\hline Age (in years) & 44.65 & 14.86 \\
\hline
\end{tabular}

Table 3: Cross tabulation for risk of malignancy index (RMI).

\begin{tabular}{|c|c|c|c|c|}
\hline & \multicolumn{3}{|l|}{ Cancer } \\
\hline & & Yes & No & Total \\
\hline \multirow{3}{*}{ RMI } & Positive & 21 (a) & $14(b)$ & $35(a+b)$ \\
\hline & Negative & $6(c)$ & $58(d)$ & $64(c+d)$ \\
\hline & Total & $27(a+c)$ & $72(b+d)$ & 99 (Total) \\
\hline
\end{tabular}

Table 4: Summary measures for risk of malignancy index (RMI).

\begin{tabular}{|l|l|l|l|l|}
\hline Statistics & Formula & Calculation & Value & $95 \%$ CI \\
\hline Prevalence & $($ Disease / Total) $* 100$ & $(27 / 99) \times 100$ & $27.3 \%$ & $18.8-37.1 \%$ \\
\hline Sensitivity & $(\mathrm{a} / \mathrm{a}+\mathrm{c}) * 100$ & $(21 / 27) \times 100$ & $77.8 \%$ & $57.7-91.4 \%$ \\
\hline Specificity & $(\mathrm{d} / \mathrm{b}+\mathrm{d}) * 100$ & $(58 / 72) \times 100$ & $80.6 \%$ & $69.5-88.9 \%$ \\
\hline Positive Predicted Value & $(\mathrm{a} / \mathrm{a}+\mathrm{b}) * 100$ & $(21 / 35) \times 100$ & $60 \%$ & $42.1-76.1 \%$ \\
\hline Negative Predicted Value & $(\mathrm{d} / \mathrm{c}+\mathrm{d}) * 100$ & $(58 / 64) \times 100$ & $90.6 \%$ & $80.7-96.5 \%$ \\
\hline Positive Likelihood ratio & Sensitivity / 1-Specificity & $0.778 / 1-0.806$ & 4 & $2.4-6.67 \%$ \\
\hline Negative Likelihood ratio & 1-Sensitivity/Specificity & $1-0.778 / 0.806$ & 0.28 & $0.13-0.56 \%$ \\
\hline Accuracy & $(\mathrm{a}+\mathrm{d}) /$ Total & $(21+58) / 99$ & $79.8 \%$ & $70.5-87.2 \%$ \\
\hline
\end{tabular}

Table 5: Cross tabulation for IOTA ADNEX model.

\begin{tabular}{|l|l|l|l|l|}
\hline \multicolumn{2}{|c|}{ Cancer } & Yes & No & Total \\
\hline \multirow{2}{*}{ IOTA ADNEX } & Positive & $21(\mathrm{a})$ & $0(\mathrm{~b})$ & $21(\mathrm{a}+\mathrm{b})$ \\
\cline { 2 - 5 } & Negative & $7(\mathrm{c})$ & $71(\mathrm{~d})$ & $78(\mathrm{c}+\mathrm{d})$ \\
\hline & Total & $28(\mathrm{a}+\mathrm{c})$ & $71(\mathrm{~b}+\mathrm{d})$ & $99($ Total $)$ \\
\hline
\end{tabular}

Table 6: Summary measures for IOTA ADNEX model.

\begin{tabular}{|l|l|l|l|l|}
\hline Statistics & Formula & Calculation & Value & $95 \%$ CI \\
\hline Prevalence & $($ Disease / Total $) * 100$ & $(28 / 99) \times 100$ & $28.3 \%$ & $19.7-38.2 \%$ \\
\hline Sensitivity & $(\mathrm{a} / \mathrm{a}+\mathrm{c}) * 100$ & $(21 / 28) \times 100$ & $75 \%$ & $55.1-89.3 \%$ \\
\hline Specificity & $(\mathrm{d} / \mathrm{b}+\mathrm{d}) * 100$ & $(71 / 71) \times 100$ & $100 \%$ & $94.9-100 \%$ \\
\hline Positive predicted value & $(\mathrm{a} / \mathrm{a}+\mathrm{b}) * 100$ & $(21 / 21) \times 100$ & $100 \%$ & $83.9-100 \%$ \\
\hline Negative predicted value & $(\mathrm{d} / \mathrm{c}+\mathrm{d}) * 100$ & $(71 / 78) \times 100$ & $91 \%$ & $82.4-96.3 \%$ \\
\hline Positive likelihood ratio & Sensitivity / 1-Specificity & $0.75 / 1-1$ & Infinity & - \\
\hline Negative likelihood ratio & 1-Sensitivity/Specificity & $1-0.75 / 1$ & 0.25 & $0.13-0.47 \%$ \\
\hline Accuracy & $(\mathrm{a}+\mathrm{d}) /$ Total & $(21+71) / 99$ & $\%$ & $86-97.1 \%$ \\
\hline
\end{tabular}

The RMI-1 model demonstrated a sensitivity of $77.8 \%$; specificity of $80.6 \%$; Positive Predictive value (PPV) 60\%; Negative Predictive Value (NPV) 90.6\%; Positive Likelihood ratio of 4, a Negative Likelihood Ratio of 2.5 and an accuracy of $79.8 \%$ in present study population
(Table 4). Again, using Histopathology as the gold standard for diagnosing malignancy the IOTA ADNEX Model had 21 true positives (a), 0 false positives(b) ,7 true negatives (c) and 71 false negatives (d) Table 5. The ADNEX model demonstrated a sensitivity of $75 \%$; 
specificity 100\%; Positive Predictive Value (PPV) 100\%; Negative Predictive Value (NPV) 91\%; Positive Likelihood ratio of infinity, a negative likelihood ratio of 2.8 and an accuracy of $92.9 \%$ in present study population (Table 6).

\section{DISCUSSION}

An Area under Curve (AUC) value of 0.978 (p value $<0.001)$ shows that IOTAADNEX has a very good predictive ability to discriminate benign from malignant adnexal masses

The overall findings demonstrated that the ADNEX Model is a better diagnostic tool as compared to RMI 1 and can help triage and counsel patients better . At $a \geq 29$ cut off the specificity of the ADNEX Model is remarkably higher than the RMI and the sensitivity of both the models is quite comparable $(75 \%$ and $77.8 \%$ respectively) Other Studies assessing the reliability of the ADNEX Model have also found that this model has a high performance when discriminating between benign and malignant adnexal masses. ${ }^{12,13}$

In present study using the cut off of 29 for the ADNEX model gives a $100 \%$ specificity and is much higher than that of RMI -1 (Specificity 80\% CI $70-89$ ). Clinically this means that when an ADNEX score is < $29 \%$ authors would confidently go for surgery at our centre and a referral to an oncology centre would not be required. In terms of sensitivity the RMI and ADNEX model are almost similar $(77.8 \%$ and $75 \%$ respectively). These findings could be extrapolated to other populations

Positive and negative likelihood ratios are also independent of disease prevalence. A positive likelihood ratio of more than 10 is the best indicator of ruling in the disease. ${ }^{9,10}$ In present study the ADNEX had a positive likelihood ratio of infinity when compared with 4 of the RMI which indicates that an ADNEX score of $\geq 29$ is the best indicator of malignancy in a woman with an adnexal mass, necessitating referral to an oncology centre. The Negative likelihood ratio: for ruling out malignancy (when the score is <29), although not ideal $(<0.1)$, is slightly better for the ADNEX model $(0.25)$ than for RMI $1(0.29)$.

The positive and the negative predictive values are dependent on disease prevalence and so these values from one study population cannot be extrapolated to other situations. Nevertheless, they can be used for counseling the woman and her relatives. In this study the positive predictive value of the ADNEX is $100 \%$ whereas that of the RMI is only $60 \%$. So, a woman with an adnexal mass having an IOTA score $\geq 29$ can be counseled for management in an oncology centre.

The negative predictive value of both the tools are quite high, as well as similar (ADNEX 91\% and RMI 90.\%) This means that a mass which has an ADNEX score of
$<29$ and an RMI score of $<200$ has a $90-91 \%$ chance of being benign. It has been seen in other studies that have used RMI as a diagnostic tool that endometriosis gives rise to a lot of false positive results which leads to decrease in the specificity of RMI 1 . This study shows that using the IOTA can successfully overcome the specificity problem (eliminating the false positives ).

Since the sensitivity still remains an issue with the IOTA ADNEX Model authors analyzed the 7 false negatives cases. Two were borderline tumors, one was a metastatic tumor from the stomach, 4 were unilocularmucinous cystadenocarcinomas with one to two papillary projections with a total diameter ranging from 7-10 and all of these four did not have an elevated CA-125 level.

These 4 mucinous cystadenocarcinomas most probably belonged to the that subgroup of mucinous ovarian malignancies which do not secrete CA-125 and instead are found to have an elevated CA 19.9. Incorporating CA 19.9 into the IOTA model may make a difference to the sensitivity. However more studies need to be conducted on this subgroup of cases to justify including this parameter.

\section{CONCLUSION}

Adding tumor specific sonographic parameters has made the IOTA ADNEX Model a better diagnostic tool to assess the nature of an adnexal mass and it outperforms the RMI 1 on almost all tests of diagnostic accuracy.

When it comes to counselling individual patients since the ADNEX and RMI have a high Negative predictive value, either an ADNEX value of $<20$ or an RMI value of $<200$ or both together can be used to reassure the patient and her relatives about the benignity of the mass.

Since the positive predictive value of the IOTA is $100 \%$ an IOTA value of $\geq 29$ would help in counselling the patient and her relatives for immediate referral and request the referral centre for prioritizing the treatment to obtain optimal results.

Because ADNEX model has a much higher specificity it has helped in excluding many false positives due to endometriosis.

The sensitivity is still not ideal probably because border line and a subgroup of uniloculated, relatively small mucinous tumours, with one or two papillary projections were missed (false negatives). Addition of CA 19.9 which is found elevated in this subgroup of mucinous tumors may increase the sensitivity of this model.

Funding: No funding sources Conflict of interest: None declared

Ethical approval: The study was approved by the Institutional Ethics Committee 


\section{REFERENCES}

1. Gillis C, Hole D, Still RM, Davis J, Kaye SB. Medical audit, cancer registration, and survival in ovarian cancer. The Lancet. 1991;337(8741):611-2.

2. Eisenkop SM, Spirtos NM, Montag TW, Nalick RH, Wang HJ. The impact of subspecialty training on the management of advanced ovarian cancer. Gynecol Oncol. 1992;47(2):203-9.

3. Kehoe S, Powell J, Wilson S, Woodman C. The influence of the operating surgeon's specialisation on patient survival in ovarian carcinoma. Brit J Cancer. 1994;70(5):1014.

4. Goff BA, Mandel LS, Drescher CW, Urban N, Gough S, Schurman KM, et al. Development of an ovarian cancer symptom index: Possibilities for earlier detection. Cancer. 2007;109(2):221-7.

5. Jacobs I, Oram D, Fairbanks J, Turner J, Frost C, Grudzinskas JG. A risk of malignancy index incorporating CA-125, ultrasound and menopausal status for the accurate preoperative diagnosis of ovarian cancer. Br J ObstetGynaecol 1990;97:922-9.

6. Irwig L, Bossuyt P, Glasziou P, Gatsonis C, Lijmer J. Designing studies to ensure that estimates of test accuracy are transferable. BMJ. 2002;324(7338):669-71.

7. Raslich MA. Markert RJ, Stutes SA. Selecting and interpreting diagnostic tests. Biochemia Medica. 2007;17(2):139-270.

8. Bossuyt PM, Reitsma JB, Bruns DE, Gatsonis CA, Glasziou PP, Irwig LM, et al. The STARD statement for reporting studies of diagnostic accuracy: explanation and elaboration. Clin Chem 2003;49(5):7-18.

9. Wilczynski NL. Quality of reporting of diagnostic accuracy studies: no change since STARD statement publication--before-and-after study. Radiol. 2008;248(3):817-23.

10. Obeidat B, Amarin Z, Latimer J, Crawford R. Risk of malignancy index in the preoperative evaluation of pelvic masses. Int $\mathbf{J}$ Gynecol Obstetrics. 2004;85(3):255-8.

11. Yamamoto Y, Yamada R, Oguri H, Maeda N, Fukaya T. Comparison of four malignancy risk indices in the preoperative evaluation of patients with pelvic masses. Europe J Obstet Gynecol Reproduct Biol. 2009;144(2):163-7,

12. Van den Akker PA, Aalders AL, Snijders MP, Kluivers KB, Samlal RA, Vollebergh JH, et al. Evaluation of the risk of malignancy index in daily clinical management of adnexal masses. Gynecologic oncology. 2010;116(3):384-8.

13. Manjunath A, Sujatha K, Vani R. Comparison of three risk of malignancy indices in evaluation of pelvic masses. Gynecol Oncol. 2001;81(2):225-9.

Cite this article as: Ray A, Divya S, Guru BNK, Ramaswamy AS, Kumar B. Diagnostic accuracy of the risk of malignancy index 1 compared to the more recent IOTA ADNEX model in discriminating benign from malignant adnexal masses: a multicentric study. Int J Reprod Contracept Obstet Gynecol 2019;8:1001-10. 


\title{
$\underline{\text { Appendix } 1}$
}

\author{
Consent Form
}

Dear Participant,

This is a study on ovarian tumors being done at 3 medical colleges. As per this study all women like you who have an ovarian tumor and are about to undergo surgery will be included in this study. As a part of this study, data about the sonographic findings and blood test levels of women like you with ovarian tumors will be collected. The information gathered will not allow identification of you as an individual and will only be used for scientific purposes

Yours,

Dr. Amita Ray

Principal Researcher

Email: dramitarays@ @ediffmail.com

Phone 9652068021

I ............. give my consent for participation in this educational project. I understand that the data collected from the Questionnaire will be used for research purposes

\section{Appendix 2}

Risk of Malignancy Index - 1 (Jacobs et al. 1990)

The Risk of Malignancy Index was calculated by

$\mathrm{U} \times \mathrm{M}$ x CA-125, where a total ultrasound score of 0 made $\mathrm{U}=0$, a score of 1 made $\mathrm{U}=1$, and a score of $\geq 2$ made $U=3$; premenopausal status made $\mathrm{M}=1$ and postmenopausal $\mathrm{M}=3$. The serum level of CA125 was applied directly to the calculation. The cut off level was selected at 200 above which was considered to indicate malignancy.

\section{Appendix 3: IOTA ADNEX variables}

Age in years (Range 14-100yrs)

Oncology Centre (Yes\No)

Maximum diameter of the lesion in mm (Range $8-400 \mathrm{~mm}$ )

Maximum diameter of the solid part in $\mathrm{mm}(0$ or $\geq 3 \mathrm{~mm})$

More than 10 locules (Yes\No)

Papillary projections (none, one ,two, three or more than 3)

Acoustic shadowing (Yes\No)

Ascites (Yes\No)

CA-125 ( Range 1-30,000 U\mL)

An on line calculator is available at

https://www.iotagroup.org/sites/default/files/adnexmodel/IOTA\%20\%20ADNEX\%20model.htm 


\section{Appendix 4: ROC curve and the coordinates of the curve}

\begin{tabular}{|c|c|c|}
\hline IOTA percentage & Sensitivity & 1 - Specificity \\
\hline-.2000 & 1.000 & 1.000 \\
\hline .8500 & 1.000 & 0.986 \\
\hline 1.0000 & 1.000 & 0.930 \\
\hline 1.1000 & 1.000 & 0.915 \\
\hline 1.1500 & 1.000 & 0.817 \\
\hline 1.2500 & 1.000 & 0.746 \\
\hline 1.4000 & 1.000 & 0.690 \\
\hline 1.5500 & 1.000 & 0.606 \\
\hline 1.6500 & 1.000 & 0.563 \\
\hline 1.7500 & 1.000 & 0.507 \\
\hline 1.8000 & 0.964 & 0.507 \\
\hline 1.8500 & 0.964 & 0.479 \\
\hline 1.9500 & 0.964 & 0.408 \\
\hline 2.0500 & 0.964 & 0.394 \\
\hline 2.1500 & 0.964 & 0.338 \\
\hline 2.2000 & 0.964 & 0.282 \\
\hline 2.2500 & 0.964 & 0.254 \\
\hline 2.3500 & 0.964 & 0.225 \\
\hline 2.4500 & 0.964 & 0.197 \\
\hline 2.6500 & 0.964 & 0.169 \\
\hline 2.8500 & 0.964 & 0.141 \\
\hline 3.0000 & 0.964 & 0.127 \\
\hline 3.1500 & 0.964 & 0.113 \\
\hline 3.4000 & 0.964 & 0.085 \\
\hline 3.6500 & 0.964 & 0.070 \\
\hline 3.7000 & 0.964 & 0.056 \\
\hline 3.8000 & 0.964 & 0.042 \\
\hline 4.1000 & 0.964 & 0.028 \\
\hline 4.9000 & 0.929 & 0.028 \\
\hline 5.5500 & 0.929 & 0.014 \\
\hline 7.5000 & 0.893 & 0.014 \\
\hline 11.1500 & 0.857 & 0.014 \\
\hline 15.3000 & 0.821 & 0.014 \\
\hline 19.4500 & 0.786 & 0.014 \\
\hline 23.3000 & 0.750 & 0.014 \\
\hline 29.9500 & 0.750 & 0.000 \\
\hline 40.3500 & 0.714 & 0.000 \\
\hline 51.4500 & 0.679 & 0.000 \\
\hline 57.6500 & 0.643 & 0.000 \\
\hline 67.1500 & 0.607 & 0.000 \\
\hline 77.8000 & 0.571 & 0.000 \\
\hline 81.0500 & 0.536 & 0.000 \\
\hline 82.7000 & 0.500 & 0.000 \\
\hline 85.8500 & 0.464 & 0.000 \\
\hline 88.7000 & 0.429 & 0.000 \\
\hline 89.7500 & 0.393 & 0.000 \\
\hline 92.8500 & 0.357 & 0.000 \\
\hline 95.3000 & 0.321 & 0.000 \\
\hline 96.7000 & 0.286 & 0.000 \\
\hline 98.2500 & 0.250 & 0.000 \\
\hline 98.7500 & 0.214 & 0.000 \\
\hline 98.9500 & 0.179 & 0.000 \\
\hline 99.0500 & 0.143 & 0.000 \\
\hline 99.3500 & 0.071 & 0.000 \\
\hline 99.7500 & 0.036 & 0.000 \\
\hline 100.9000 & 0.000 & 0.000 \\
\hline
\end{tabular}




\section{Appendix 4: ROC curve and the coordinates of the curve}

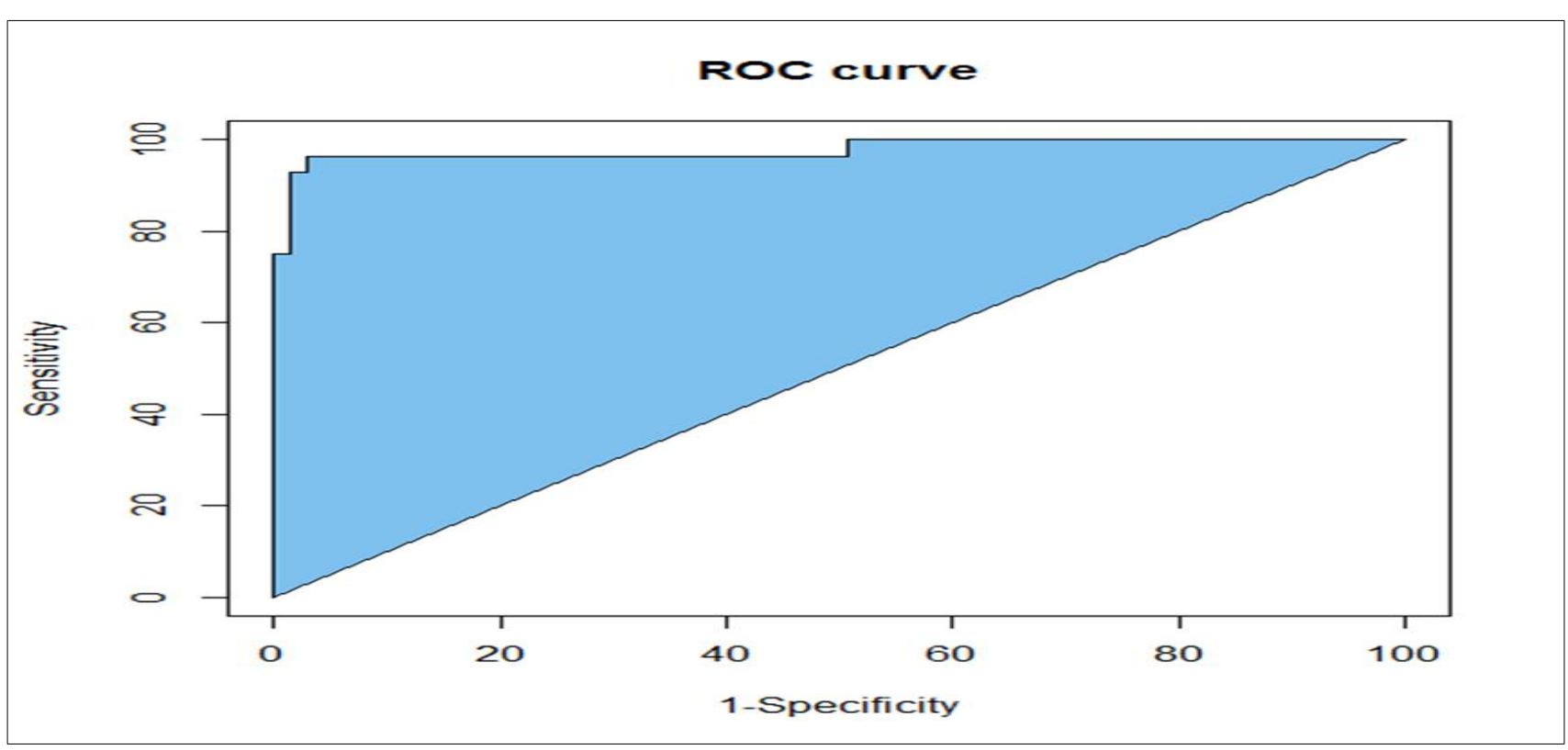




\section{Appendix 5: STARD guidelines}

\section{Present study}

\section{Tests of diagnostic accuracy}

Sensitivity, Specificity, Likelihood Ratios, Positive and Negative Pred

\begin{tabular}{|l|}
\hline Abstract \\
\hline Abstract given as per STARD Guidelines \\
\hline Introduction \\
Introduction as per STARD Guidelines \\
To establish a cut off for the IOTA ADNEX model \\
in our population 2) Compare the ability of RMI1 \\
and ADNEX IOTA Model to differentiate between \\
benign and malignant adnexal masses \\
Methods
\end{tabular}

Study design: Data collection planned before index test (prospective study)

\section{Participants}

All women with a diagnosis of an adnexal mass scheduled for surgery

Three centers from 2017-2018

Consecutive

\section{Test methods}

Risk of Malignancy Index RMI (detailed in appendix) IOTA ADNEX Model (detailed in appendix)

Reference standard histopathology

Histopathology golden and final diagnosis alternatives do not exist

Cut off for RMI as specified by RCOG is 200

The cut off for the ADNEX IOTA Model was calculated using an ROC

No cut offs in Histopathology it would be either yes for malignancy and no for benign

No

No

\section{Analysis}

Statistical software for calculation of al the tests specified above

There were no indeterminate tests

There were no missing data

No variability

\section{STARD guidelines}

Identification as a study of diagnostic accuracy using at least one measure of accuracy (such as sensitivity, specificity, predictive values, or AUC)

Structured summary of study design, methods, results, and conclusions (for specific guidance, see STARD for Abstracts)

Scientific and clinical background, including the intended use and clinical role of the index test

Study objectives and hypotheses

Whether data collection was planned before the index test and reference standard were performed (prospective study) or after (retrospective study)

Eligibility criteria

On what basis potentially eligible participants were identified (such as symptoms, results from previous tests, inclusion in registry)

Where and when potentially eligible participants were identified (setting, location and dates)

Whether participants formed a consecutive, random or convenience series

Index test, in sufficient detail to allow replication

Reference standard, in sufficient detail to allow replication Rationale for choosing the reference standard (if alternatives exist)

Definition of and rationale for test positivity cut-offs or result categories of the index test

Definition of and rationale for test positivity cut-offs or result categories of the reference standard, distinguishing prespecified from exploratory

Whether clinical information and reference standard results were available to the performers/readers of the index test Whether clinical information and index test results were available to the assessors of the reference standard

Methods for estimating or comparing measures of diagnostic accuracy

How indeterminate index test or reference standard results were handled

How missing data on the index test and reference standard were handled

Any analyses of variability in diagnostic accuracy, distinguishing pre-specified from exploratory

Intended sample size and how it was determined 


\section{Results}

\section{Participants}

Specified in results section

Not assessed

Baseline demographic and clinical characteristics of participants

Endometriosis

Distribution of severity of disease in those with the target condition

Time interval mean 7 days No clinical interventions Distribution of alternative diagnoses in those without the target condition

\section{in between}

Time interval and any clinical interventions between index test

\section{Test results}

Specified in results section and reference standard

Specified in results section

\section{Nil}

\section{Discussion}

Specified in discussion

Specified in discussion
Cross tabulation of the index test results (or their distribution) by the results of the reference standard

Estimates of diagnostic accuracy and their precision (such as 95\% confidence intervals)

Any adverse events from performing the index test or the reference standard

Study limitations, including sources of potential bias, statistical uncertainty, and generalisability

Implications for practice, including the intended use and clinical role of the index test 